REVISTA ANDALUZA DE ANTROPOLOGÍA

NÚMERO 14: IRRUPCIONES FEMINISTAS. PROBLEMÁTICAS EPISTEMOLÓGICAS Y POLÍTICAS. FEMINIST EMERGENCES. EPISTEMOLOGICAL AND POLITICAL ISSUES

MARZO DE 2018

ISSN 2174-6796

[pp. 7-22]

http://dx.doi.org/10.12795/RAA.2018.14.02

\title{
CONCEPTUALIZAR LA VIOLENCIA CONTRA LAS MUJERES: ¿UNA REFLEXIÓN EPISTEMOLÓGICA PENDIENTE?
}

\section{CONCEPTUALIZING VIOLENCE AGAINST WOMEN: AN EPISTEMOLOGICAL REFLECTION PENDING?}

\section{Carme Adán \\ IES Politécnico de Vigo}

\section{Resumen.}

Las epistemologías feministas tienen entre sus objetivos fundamentales contribuir a un cambio real en el conocimiento que comporte vías de intervención desde una perspectiva que favorezca a las mujeres. En este sentido, la objetividad lejos de ser eliminada de la agenda feminista, pasa a ocupar uno de los primeros planos. Son las epistemólogas feministas las más interesadas en mostrar el carácter erróneo de concebir la objetividad en términos de neutralidad valorativa. En este contexto, la objetividad es sometida a una reconfiguración introduciendo en el debate la dimensión ético-política. En el ámbito de la eliminación de la violencia contra las mujeres el feminismo ha jugado un papel fundamental. Determinar qué es y en qué consiste la violencia contra las mujeres ha sido y es en la actualidad una fase necesaria para poder intervenir y, también, para poder prevenirla. Las categorías utilizadas por diferentes leyes y tratados son fruto del trabajo de reconceptualización realizado por la teoría feminista. Por lo tanto, consideramos de interés en primer lugar, revisar desde la epistemología feminista, en concreto haciendo uso de las nociones de objetividad enunciadas por Sandra Harding y Dona Haraway, las categorías que subyacen diferentes documentos legislativos. En segundo lugar, 
revisaremos la violencia contra las mujeres introduciendo el concepto de situación y la noción de libertad.

Palabras clave: epistemología feminista, género, experiencia de las mujeres, violencia contra las mujeres, violencia de género, feminicidio

\title{
Abstract.
}

Feminist epistemologies try to fundamentally contribute to an actual change in knowledge that involves ways to intervene in society from a women-centric perspective. In this sense, objectivity is far from being removed from the feminist agenda. Quite the opposite, feminist epistemologists are keen on showing how wrong it is to think of objectivity in terms of valuing neutrality. In this context, objectivity is subjected to a reconfiguration, introducing an ethical and political dimension to the debate. In the field of the eradication of violence against women, feminism has played a fundamental role. Determining what it is and what gender violence is about is a necessary step to intervene and, also, to prevent. The categories used in different laws and treaties are the outcome of the reconceptualization produced by feminist theory. Therefore, we would like to first review, from a feminist epistemology, and using the notions of objectivity discussed by Helen Longino, Sandra Harding and Dona Haraway, the categories underlying different legal texts. Secondly, we will introduce the concept of situation in order to review violence against women from feminst epistemoloy and liberty notion.

Key words: feminist epistemology, gender, women experience, violence against women, gender violence, feminicide

\begin{abstract}
"Ahora que estoy muerta lo sé todo", esperaba poder decir, pero, como tantos otros de mis deseos, éste no se hizo realidad. Sólo sé unas cuantas patrañas que antes no sabía. Huelga decir que la muerte es un precio demasiado alto para la satisfacción de la curiosidad."
\end{abstract}

Margaret Atwood

La búsqueda de conocimiento por parte de Penélope en su bajada al Hades da inicio a otra versión de la Odisea. La escritora Margaret Atwood (2005) restituye la voz de Penélope y las doce criadas. Ellas cuentan la otra historia, la de su asesinato y silencio. Desean saber, desean justicia. 
Conocer es un acto prohibido para las mujeres. El deseo de saber es una expresión de resistencia, de insubordinación. Saber quién impuso las leyes, las palabras, los silencios. Saber quién decide el orden de las cosas, quién decide sobre la vida yla muerte. Las mujeres, apartadas contra su voluntad de las Academias, de los Liceos, de las Universidades, transformaron sus ansias de conocimiento en la fuerza necesaria para derrumbar las puertas y los muros de los prohibidos espacios de saber. Su esfuerzo no solo las dotó del reconocimiento como sujetos cognoscentes sino que fue decisivo para cuestionar el carácter neutral de la episteme y mostrar su naturaleza social. Vincular ciencia y valores es una tarea, que lejos de invalidar el conocimiento, proporciona mayor capacidad para contribuir al bienestar humano y a diseñar visiones menos dominadoras de la realidad. Ahondar en la comprensión del conocimiento como una práctica social nos obliga a reconocer que las preguntas sobre el saber van más allá de una voluntad de conocimiento y se enraízan en la voluntad de poder y control.

El feminismo es una revolución sostenida en el tiempo. A pesar del olvido perpetrado por la historia oficial, es posible, en todos los tiempos, encontrar mujeres que han desafiado las normas de sus correspondientes tribus y han conquistados espacios de libertad para ellas y otras mujeres. Esta revolución también ha impregnado la propia construcción del conocimiento. De hecho, la teoría feminista ha considerado fundamental tres tareas a este respecto, a saber, mostrar la importancia de los saberes tradicionales elaborados por las mujeres y excluidos de las academias; recuperar las pioneras de todos los tiempos que practicaron la insumisión cognitiva y, por último, cuestionar la propia naturaleza del conocimiento, entre otros aspectos su supuesta neutralidad y objetividad.

Hace ya varias décadas que la teoría feminista, junto a otros estudios sociales de la ciencia $^{1}$, se propuso como empresa prioritaria la revisión de los valores sociales y los sesgos de género que permean el altar más sagrado de la neutralidad epistémica. La crítica feminista del conocimiento ha llegado al punto de cuestionar la propia naturaleza androcéntrica del mismo, tal como se ha desarrollado y trasmitido por el saber oficial. Al realizar este esfuerzo de análisis y crítica, el feminismo ha vivido, y vive, en sus propias carnes una de las mayores contradicciones, la que tan bien resumía Audre Lorde en su famosa conferencia "Las herramientas del amo nunca desmontan la casa del amo" (2004). Crear un conocimiento no patriarcal partiendo de la revisión y resignificación del mismo podemos intuir que es un reto para nada fácil. Analizar qué conceptos recibidos de la tradición son adecuados, cuales están impregnados y, en qué grado, de los valores sexistas de la sociedad en la que fueron pensados o crear nuevos marcos cognitivos y nuevos paradigmas requiere de un importante trabajo y corpus teórico por parte de las pensadoras feministas. Dentro de este corpus teórico en constante transformación

1. Existe una amplia bibliografía sobre la relación entre la epistemología feminista y los estudios CTS -Ciencia, Tecnología y Sociedad-, sobre temas y periodización de los mismos puede verse Echeverría (1999). 
se ha denominado epistemología feminista a los estudios y reflexiones que abordan las cuestiones referidas a la naturaleza, componentes y versiones más adecuadas del conocimiento.

Este texto pretende acercarse, desde una óptica epistemológica, a un concepto fundamental para comprender nuestra sociedad y también la evolución del feminismo, el concepto de violencia de género. Huelga decir que conocer es el primer paso en el abordaje de la violencia contra las mujeres. De hecho, es habitual establecer tres fases, en concreto, conocer, intervenir y erradicar (Lagarde, 2008). Al ser enunciadas de forma sucesiva podemos dar la errónea impresión de que forman un proceso continuado o lineal, cuando en realidad después de años de intervención comenzamos a conocer la violencia de género, o hablando con mayor precisión, tenemos marcos interpretativos que muestran las muchas variables de un fenómeno tan complejo.

La violencia contra las mujeres tiene tras de sí una historia de naturalización e invisibilización que la normalizó a los ojos de la sociedad, el derecho, la ciencia y el diseño de las políticas públicas. Por lo tanto, el primer mérito del feminismo fue desvelar su carácter social, dotar de términos y conceptos una realidad que se ha ocultado en el interior de las casas. Conseguir que la violencia contra las mujeres formara parte de las leyes y de las preocupaciones del Estado es un gran logro. Desde nuestra óptica, ahora es necesario profundizar en el marco interpretativo más idóneo que permita entender cómo la violencia se ha convertido en un elemento central de nuestra sociedad y de la discriminación que viven las mujeres.

La antropóloga Rita Segato defiende que entender las formas de la violencia de género hoy es entender lo que atraviesa la sociedad como un todo (2016). Consideramos de interés esta idea, a modo de punto de apoyo, para poder cambiar nuestra mirada. Puede que no sea interrogando a la sociedad y sus asimetrías de género como comprendamos qué es la violencia. Cambiemos la mirada y pongamos todos nuestros artilugios visuales sobre la violencia contra las mujeres para que se nos vuelva inteligible la reproducción de la desigualdad que el heteropatriarcado imprime en la sociedad. Para esta tarea podemos hacer uso de las aportaciones de la epistemología feminista, que permite analizar qué valores y qué consensos se han establecido en la construcción de los diferentes conceptos que intentan delimitar y categorizar la violencia contra las mujeres.

\section{UNA PINCELADA DE EPISTEMOLOGÍA FEMINISTA}

La teoría feminista ha mostrado una gran capacidad para cuestionar y transformar el conocimiento heredado. Aplicar el adjetivo de feminista a la "epistemología" requiere algo más que hacer explícito desde donde se investiga, implica un cuestionamiento de la naturaleza misma del conocimiento. La epistemológica feminista centra sus intereses en reflexionar respecto de los elementos centrales sobre los que se ha establecido qué 
es conocimiento, tales como sujeto, objeto, verdad y objetividad buscando como meta principal remover los obstáculos que han producido y reproducido la discriminación e infravaloración de las mujeres y lo femenino en su conjunto (Adán, 2006; Harding, 1986; Longino, 1990; Pérez Sedeño, 1999).

No es el propósito de este texto profundizar en la importancia que conlleva analizar el papel de la objetividad y la neutralidad en la construcción del conocimiento en general, y de la ciencia en particular. Sin embargo, podemos destacar que son dos conceptos fundamentales sobre los que se asienta en gran medida lo que entendemos por verdad y realidad. Por lo tanto, nuestras visones del mundo. No es propiedad exclusiva de la epistemología feminista cuestionar el carácter neutral del conocimiento ni someter a debate que es la objetividad y de que depende, no obstante, su contribución ha sido determinante ya que ha puesto de manifiesto el carácter androcéntrico del conocimiento desde la raíz misma de su formulación. Así, para las autoras de las corrientes más representativas de la epistemología feminista redefinir la objetividad es una empresa fundamental.

Estas corrientes son habitualmente reconocidas como tres, a saber, el empirismo feminista, la teoría del punto de vista feminista y la propuesta postmoderna. Solo a modo de breve puntualización debemos comentar que comparten como punto de partida el reconocimiento del pluralismo y el papel central de los valores en el conocimiento, una redefinición del sujeto cognoscente en función de las comunidades epistémicas y también la huida del relativismo, tan poco aliado con los intereses de las mujeres y los grupos oprimidos. Como autoras destacadas del empirismo feminista, la teoría del punto de vista feminista y la posmodernidad feminista nos referiremos a Helen Longino, Sandra Harding y Donna Haraway respectivamente.

Para Helen Longino (1990), autora que comparte con Lynn Nelson (1990) la difícil tarea de revisar el empirismo filosófico desde la perspectiva de las mujeres, la objetividad no debe implicar nunca la neutralidad en valores y adquiere un sentido de consenso comunitario. Las comunidades son los espacios donde se produce el conocimiento fruto de dicho consenso. La tesis central de esta autora es que la neutralidad política de la ciencia es una posición política en sí misma y no metodológica ni espistemológica. Asumir el papel de los valores en la empresa epistémica favorece teorías que mejoran las condiciones de vida y tienden a la eliminación de las desigualdades.

Sandra Harding (1986) es la autora más representativa de la teoría del punto de vista feminista y una de las pensadoras más destacadas en el ámbito de la epistemología. Ella acuñó el término objetividad fuerte en un intento por huir de la mistificación de la objetividad y no caer en el relativismo. Así considera que la objetividad fuerte debe ser entendida como un método que detecte los valores e intereses que constituyen los proyectos científicos, las diferencias que existen entre valores e intereses que amplían o 
limitan la imagen del mundo y las relaciones humanas y, por último, los acuerdos a los que se llegue deben primar el consenso entre las comunidades epistémicas (1995).

Donna Haraway (1995), autora conocida por su propuesta del cyborg como metáfora para pensar el sujeto feminista, representa la apuesta epistemológica desde una óptica denominada posmoderna. Haraway defiende una transformación del conocimiento con fines ético-políticos que cristaliza en una propuesta de descripciones mejores del mundo. Define la objetividad en los siguientes términos: "yo quisiera una doctrina de la objetividad encarnada que acomode proyectos de ciencia feminista paradójicos y críticos: la objetividad feminista significa, sencillamente, conocimientos situados" (Haraway 1995: 324). La estrategia para construir una nueva noción de objetividad reside en la propia mirada situada, en el propio reconocimiento de la parcialidad se configura una visión mejor del mundo (1997).

Aunque sea de un modo muy esquemático podemos observar que tanto Longino como Harding o, incluso, Haraway proponen diferentes versiones de la objetividad como compromiso feminista. Estas tres autoras, como todas las pensadoras que dedican espacio a la epistemología, someten a análisis crítico el concepto de objetividad. Longino retoma la noción de contexto y comunidades epistémicas para destacar el papel de los valores en su formulación, Harding habla de una objetividad fuerte entendida desde la puesta en común de la experiencia de las mujeres y Haraway da forma a la noción de situación a través de la objetividad. Si realizamos una puesta en común de estas tres formas de acercarnos a la construcción del conocimiento, la noción de situación es un aspecto central.

Como ya hemos defendido en otros trabajos la noción de situación da forma al trasfondo común que cada mujer vive o tiene experiencia de forma individual. Este concepto que evita los esencialismo reduccionistas se convierte en una categoría que "designa la fusión entre la ficción política del sujeto mujeres y la materialidad concreta del cuerpo de las mujeres. Esta noción permite comprender la dimensión que la contextualidad del conocimiento adquiere para el feminismo" (Adán, 2006:312). Esta óptica puede ser de utilidad para acercarnos a la violencia contra las mujeres desde la epistemología feminista. Algunos desarrollos críticos del Derecho han apuntado esta línea de investigación (Torres, 2016). Indagar en los valores que atraviesan los diferentes conceptos con los que se ha descrito esta situación que viven las mujeres como grupo dentro del patriarcado nos facilita, por un lado, el análisis de los valores propios de los diferentes paradigmas desde los que se ha considerado la violencia, por otro, consigue no situar a las mujeres como víctimas, sino que nos permite analizarlas desde la vulnerabilidad como trasfondo común de la estructura social que cada mujer vive en su carne . 


\title{
2.NOMBRAR, PENSAR, CONOCER LA VIOLENCIA CONTRA LAS MUJERES: UN PROCESO FLUIDO
}

\author{
"En El segundo sexo, Simone de Beuavoir sostenía que no se nace \\ mujer sino que se llega a serlo. Es indudablemente cierto que los \\ significados de la palabra se acumulan y cambian incluso en el \\ transcurso de una sola vida”.
}

Siri Hustvedt

No podemos pensar lo que no tiene nombre. No podemos conocer aquello que ni tan siquiera podemos pensar. De ahí que la violencia contra las mujeres esté presente en la historia de la humanidad bajo el patriarcado como lo natural, lo invisible, lo que ocurre sin causas. Dotar de voz a las mujeres, ayudar a nombrar, buscar palabras para el dolor ha sido un momento crucial para el feminismo. Conceptualizar la violencia contra las mujeres ha logrado dar un paso más allá, ha permitido politizarla, en el sentido filosófico de proyectarla en el espacio de lo público. Sin embargo, los conceptos, como la vida, fluyen y se adaptan. Nada es estático, la conceptualización de la violencia tampoco.

Hoy, más de dos décadas después de que los tratados internacionales utilizaran por primera vez la expresión violencia de género con el objetivo de reconocer que el origen de la violencia contra las mujeres estriba en el reparto asimétrico de poder y reconocimiento entre hombres y mujeres, necesitamos reparar en algunas preguntas sobre los caminos de futuro que debemos transitar.

Para este fin necesitamos, en primer lugar, reconocer que la incorporación de la categoría género fue un elemento decisivo que permitió destacar el carácter de construcción histórica y cultural de la violencia. Ha sido un logro colectivo del feminismo conceptualizar la violencia contra las mujeres fuera del marco de lo doméstico y lo pasional. Este proceso ha contado con fuertes resistencias por parte del saber establecido. Basta como ejemplo el informe que la RAE lanzó en 2004 en contra del término violencia de género recomendando el uso de violencia doméstica respecto del debate y aprobación de la Ley integral. La preocupación de una institución encargada de los usos de la lengua por eliminar un término en una ley decisiva para crear recursos y servicios no es una cuestión menor o colateral como con mucho acierto ha denunciado Nuria Varela (2017). La lengua no es neutral, su historia es un buen ejemplo de cómo los valores permean nuestra forma de denominar la realidad y, por ende, de pensarla. La utilización de lo doméstico y lo pasional tiene su origen en una concepción de la violencia contra las mujeres como lo natural dentro del matrimonio o del amor romántico atravesado por celos y control. No es tema de este escrito profundizar sobre los sesgos de género de las 
comunidades epistémicas de lingüistas y filólogos, aunque es evidente que son un buen ejemplo del uso político de la noción de neutralidad y objetividad.

En segundo lugar necesitamos reconocer que los logros conseguidos en la conceptualización de la violencia contra las mujeres no pueden impedirnos avanzar aunque sea problematizando alguna de nuestras propuestas previas. En la actualidad, a pesar de los muchos escritos críticos sobre la categoría de género, su uso semeja que es equiparable a mujeres, de hecho, es en la propia definición de violencia de género donde de forma reiterada ocurre esto. A pesar de que la idea ha sido clara, marcar el carácter social de la desigualdad y, por lo tanto, las denominadas asimetrías de género, el problema estriba en que es un concepto que señala más el resultado que el proceso de patriarcalización. Estamos obligadas a interpelarnos sobre nuevas nociones, o revisiones de las ya existentes de la violencia contra las mujeres con el objetivo de buscar la mejor clarificación para aportar mayores elementos a las fases de intervención y erradicación.

Comencemos por recordar un poco de la historia institucional del propio concepto de violencia de género. En la década de los ochenta la ONU, en su II Conferencia internacional sobre las mujeres, declaró, por primera vez, que la violencia que se ejerce contra las mujeres en la familia es el crimen más encubierto del mundo y planteó la importancia de visibilizar públicamente esta problemática que afecta a un gran número de mujeres. En los noventa, la Asamblea General de la ONU aprobó la «Declaración sobre la eliminación de la violencia hacia la mujer», en la que reconoce que la violencia hacia la mujer constituye una manifestación de relaciones de poder históricamente desiguales entre el hombre y la mujer, que han conducido a la dominación de la mujer y a la discriminación en su contra por parte del hombre e impedido el avance pleno de la mujer, y que la violencia contra la mujer es uno de los mecanismos sociales fundamentales por el que se fuerza a la mujer a una situación de subordinación respecto al hombre. Sin embargo, fue en 1995 cuando la Declaración de Beijing, surgida de la IV Conferencia mundial sobre la mujer, definió por primera vez la violencia contra las mujeres como violencia de género, entendiendo ésta como una construcción cultural que rige las relaciones sociales y de poder entre los sexos.

Incluir en esta declaración un concepto como el de género, nacido a la luz de la antropología por la autora Gayle Rubin (1975), fue decisivo para mostrar que las causas de la violencia contra las mujeres hay que buscarlas en las construcciones culturales sobre la que se establecen los códigos normativos y axiológicos a partir de los cuales podemos hablar de lo masculino y lo femenino en un marco de relaciones de poder asimétricas. El concepto género tiene un historia compleja y decisiva para la elaboración de una buena parte de las teorías feministas actuales. Podemos rastrear su huellas en los escritos de la pensadora feminista del siglo XVIII Wollstonecraft sobre la defensa de la socialización igualitaria de las mujeres, pero sin lugar a dudas será Simone de Beauvoir quien marcará 
con rotundidad el carácter social y aprendido de la experiencia femenina con su famosa frase "no se nace mujer, se llega a serlo" (Beauvoir, 1989:13).

La virtualidad de la utilización de la noción de género por parte de Rubin para referirse al sistema sexo/género estriba en que permite investigar las causas sociales que conducen a la jerarquización de lo masculino y femenino rechazando cualquier explicación biologicista. Quizás sea de interés reproducir su primera definición a este respecto: "Como una definición preliminar, un "sistema sexo/género" es un conjunto de disposiciones por las que una sociedad transforma la sexualidad biológica en productos de la actividad humna $y$ en los que estas necesidades sexuales transformadas son satisfechas" (Rubin, 1998,28). Una crítica común a su noción, y que compartimos, es que Rubin en su propuesta adopta las teorías de Marx, Freud y Levi-Strauss construidas desde la masculinidad abstracta desoyendo la propia experiencia de las mujeres y su vida material (Harsock, 1998).

La separación de la propia experiencia de las mujeres, concepto fundamental en la teoría del punto de vista feminista y algunas propuestas como la de Haraway, da lugar a una parte de los problemas que genera el uso del término género. Por un lado, es un término paraguas que implica un abanico de significados (Rosi Braidotti, 1999, Joan Scott, 1990). Por otro, ha permitido desarrollos posteriores (Butler, 1990) que han problematizado la propia categoría permitiendo separarla del sexo y mostrando, con ello, tanto la proliferación de géneros como el carácter de construcción cultural de ambos, género y sexo. Los desarrollos teóricos posmodernos y queer beben de estas propuestas y han desarrollado en la constante resignificación de los géneros un potente instrumento de lucha política (Platero, 2017). Por último, la categoría género ha permitido superar las reticencias que el término feminismo producía en las academias o en la sociedad en su conjunto y, así, poder ser utilizada en expresiones como perspectiva de género o violencia de género. Sin embargo, lo que en principio parecía ser una virtualidad, un uso ritual o mecánico puede implicar que el patriarcado, como estructura de poder que configura la sociedad en su conjunto quede difuminado o invisibilizado ${ }^{2}$.

En concreto y referido a la violencia contra las mujeres, que es el tema que aquí nos concierne, su uso habitual en la sociedad es sinónimo de violencia contra las mujeres. En el estado español la Ley Orgánica 1/2004, de 28 de diciembre, de Medidas de Protección Integral contra la Violencia de Género comienza su preámbulo con las siguientes palabras:

"La violencia de género no es un problema que afecte al ámbito privado. Al contrario, se manifiesta como el símbolo más brutal de la desigualdad existente

2. Como ya se ha indicado, el concepto género y otros como heteropatriarcado o violencia, tienen una historia rica y compleja. Autoras como $\mathrm{M}^{\mathrm{a}}$ Jesús Izquierdo, Paloma Uría o Raquel Osborne, entre otras, ofrecen una óptica muy interesante para ampliar el debate que planteamos en este texto, más que excede las posibilidades del mismo. 
en nuestra sociedad. Se trata de una violencia que se dirige sobre las mujeres por el hecho mismo de serlo, por ser consideradas, por sus agresores, carentes de los derechos mínimos de libertad, respeto y capacidad de decisión.”

Por lo tanto, la violencia de género queda definida como la violencia que sufren las mujeres por ser mujeres pero que tiene su origen en el aspecto social del género. Es decir, la construcción de roles de género, considerados de forma asimétrica en cuanto a su reconocimiento, es lo que se pretende enfatizar. La desigualdad adquiere un papel central en la definición que realiza la ley de la violencia de género, incluso se considera como un símbolo, o manifestación de la propia desigualdad. Este aspecto es importante y volveremos sobre el en el siguiente apartado.

El reciente Pacto de Estado en materia de violencia de género aprobado en octubre de 2017 por el Congreso de los Diputados, asume las definiciones de violencia de género propias del Convenio de Estambul y, por lo tanto, avanza la ampliación de la "violencia de género" de la siguiente forma:

"Declarar que la violencia contra las mujeres constituye una violación de los derechos humanos y una forma de discriminación, y comprende todos los actos de violencia basados en el género que implican o puedan implicar para las mujeres, daños o sufrimientos de naturaleza física, sexual, psicológica o económica, así como cualquier otra forma de violencia que afecte a las mujeres de forma desproporcionada. A estos efectos también se entenderá como violencia contra las mujeres, las amenazas de realización de dichos actos, la coacción o la privación arbitraria de libertad, en la vida pública o privada."

Como podemos observar la incorporación de esta modificación a la LO 1/2004 profundiza en la concepción de la violencia de género como una expresión de la discriminación y la desigualdad. Define textualmente que comprende todos los actos de violencia basados en el género, asumiendo, por lo tanto, que género se refiere a todo el entramado social que articula la sociedad como un todo. Definitivamente sería mucho más claro y conceptualmente más correcto afirmar que comprende todos los actos de violencia contra las mujeres fruto del heteropatriarcado, ya que lo que viven todas las mujeres por ser mujeres es una situación de vulnerabilidad heredada por ese fondo al cual nos referíamos con anterioridad. El patriarcado, en cada momento histórico, produce una situación de vulnerabilidad de las mujeres que se concretiza en la violencia contra las mujeres, la cual deviene en mecanismo eficaz para mantener la desigualdad.

Por todo lo argumentado hasta este momento, en el siguiente apartado nos plantearemos la necesidad de cambiar la pregunta. Conocer la violencia implica, no solo una perspectiva psicológica sobre la víctima, sino preguntar a quién o a qué estructuras sociales y de poder beneficia. El patriarcado se sostiene en la falta de libertad de las mujeres y sus 
reiteradas adaptaciones históricas han buscado formas para erradicar la autonomía de las mujeres en nombre del supuesto bien de estas. En este sentido ha encontrado un aliado en los procesos de construcción del conocimiento y la ciencia. Por lo tanto, las reflexiones realizadas desde el prisma de la epistemología feminista tienen su razón de ser. De hecho, sostenemos que no someter a una óptica epistemológica el concepto de violencia explica, en parte, la percepción que existe en el feminismo de que llegamos a un punto que caminamos en bucle. Esto es, proliferan las leyes y los tratados internacionales y aumenta la violencia contra las mujeres. Necesitamos un compromiso efectivo con la despatriarcalización de la política y pensar la violencia desde todos los prismas es una parte del proyecto. Finalizamos este apartado con una cita de la pensadora feminista Celia Amorós que invita a tomar esta dirección:

"El núcleo de la cuestión estribaría en determinar si el feminicido es una categoría específica o bien se la puede aplicar a todo tipo de crímenes perpetrados contra las mujeres. Siempre he afirmado que conceptualizar es politizar: en caso de la mal llamada violencia doméstica (...). En la misma línea, lo que ahora está sobre el tapete son las ventajas epistemológicas y políticas de singularizar conceptualmente el feminicidio idiosincrático (...) se impone identificar las características precisas que reviste la violencia feminicida." (Amorós, 2008:269)

\section{CAMBIEMOS LA MIRADA: SITUADAS EN LA VIOLENCIA}

$\mathrm{Al}$ acercarnos a la violencia contra las mujeres el marco de análisis que utilizamos es determinante para conseguir desnaturalizar y establecer los mecanismos sociales necesarios para erradicarla. En esta línea las contribuciones del paradigma feminista son notables y decisivas para situar la violencia fuera de la privacidad y lo doméstico. Afirma la filósofa Agra:

"Los estudios feministas nos van a aportar una visión de la complejidad de la violencia, de la violencia sistemática, como hecho y práctica social inserta en una estructura jerárquica que regula socialmente las relaciones entre hombres y mujeres, el denominado sistema sexo-género, mostrando como en la práctica la(s) violencia(s) implican carencia de derechos y afectan al estatus y al ejercicio pleno de la ciudadanía. El problema de las violencias específicas contra las mujeres no está ni en la naturaleza ni es reducible a patologías, es un problema social y político" (Agra, 2013:37).

Aquello que es un problema social y político, como establecimos en el apartado anterior, debe ser pensado en tales términos. De ahí la importancia de poder conceptualizar con precisión. Ese ha sido, no podemos negarlo, el gran esfuerzo de las filósofas feministas en las últimas décadas. Buscar categorías que nos permitan pensar las diferentes variables que componen la matriz de dominación sobre la que el heteropatriarcado ha establecido 
su vigencia a lo largo de la historia humana. Autoras como la antropóloga Rita Laura Segato, con cientos de páginas dedicadas a analizar la violencia contra las mujeres, llega a afirmar el carácter central de la violencia contra las mujeres para entender nuestra sociedad. Segato (2016) escudriña en las estructuras de las violencias feminicidas de nuestro tiempo y nos proporciona herramientas conceptuales para comprender el aumento de la violencia contra las mujeres en las sociedades modernas decoloniales. Este papel central de la violencia nos ha inspirado para aplicar la óptica epistemológica. La violencia contra las mujeres es una injusticia que nos priva de libertad. Es el dispositivo social más fuerte para limitar la libertad y reproducir las desigualdades. Así, por mucho que las leyes recojan la violencia como una manifestación de la desigualdad, la sociedad sigue ejerciéndola como una forma de control de las propias mujeres.

La violencia contra las mujeres tiene un carácter fundacional en el diseño del patriarcado contemporáneo. Patriarcado que, aliado con fuerza del capitalismo financiero y el individualismo neoliberal, conjura la apariencia de libertad que el neoliberalismo ha impuesto, haciendo uso del mantra "es lo que ellas deciden", con la violencia simbólica y real como mecanismo de dominación. La violencia contra las mujeres marca los límites de la sociedad actual. Recuerda a niñas, jóvenes y mujeres adultas que el poder se establece de forma jerárquica y desacatarlo tiene consecuencias. La cultura de la violación o la pedagogía de la crueldad son conceptos que proliferan para explicar datos aterradores ${ }^{3}$. Compartimos con muchas feministas que debemos huir de la victimización de las mujeres. No somos víctimas, no compartimos una esencia porque el problema no está en la naturaleza. El problema es social y lo que compartimos es una situación, ese trasfondo común que tematizaba de Beauvoir, que en el caso de la violencia como explica Agra (2016) toma forma a través de la vulnerabilidad. La situación que compartimos todas es la de carencia de libertad y, por lo tanto, de vulnerabilidad. Entendido este concepto de vulnerabilidad como una construcción social impuesta por las propias estructuras de dominación, la vulnerabilidad de la especie en diferentes momentos o etapas de la vida y la necesidad del cuidado es una derivada importante de este debate que excede a las posibilidades de análisis de este texto. En lo referido a cómo vive cada mujer que sufre la violencia de género su abordaje concreto desde la psicología, el derecho y las políticas públicas tampoco conciérne al debate de este texto.

En las últimas décadas la violencia contra las mujeres la hemos expresado en términos de carencia de igualdad, en concreto como la máxima expresión de la desigualdad entre hombres y mujeres. Así fue recogido en diferentes textos legislativos, como hemos indicado en el apartado anterior. Desde esta premisa entendemos la repetición, por parte

3. Según el último informe elaborado por la Agencia de los derechos fundamentales de la Unión Europea (2014) cada año en los 28 estados miembros de la UE 13 millones de mujeres sufren violencia física, 3,7 millones son violadas y 9 millones son víctimas de acoso. A lo largo de su vida 62 millones de europeas sufren violencia física o sexual, esto es, una de cada tres mujeres que viven en la UE. 
de poderes públicos y los medios de comunicación, de la idea de que el camino de la igualdad real entre hombres y mujeres conduce a la desaparición de la violencia. Sin entrar a discutir ese espacio utópico de igualdad plena, que todo apunta a que estamos a una distancia considerable, para una reflexión epistemológica como la que nos ocupa consideramos más pertinente volver sobre el concepto de libertad. La violencia contra las mujeres es el principio constitutivo de la desigualdad, no en un sentido temporal, pero si en sentido fundacional. El control sobre las mujeres se ejerce desde múltiples frentes, siendo el de la violencia uno de los más disciplinarios. Las niñas crecen en la cultura del miedo a la violencia, a la violación, al caminar solas por la calle, al acoso en el aula, en sus casas por parte de familiares y amigos... Una cultura que priva de libertad $\mathrm{y}$ es funcional al patriarcado.

Conocer la violencia contra las mujeres implica preguntarnos por las estructuras que se mantienen vigentes con su ejercicio. Las estructuras no matan, lo hacen los hombres educados en un sistema que les impone como mandato social el control, simbólico y material, sobre las mujeres. Diferenciar entre violencia contra las mujeres, violencia de género, violencia machista, violencias feminicidas no es un ejercicio semántico para los ratos libres, es dotarnos de focos que iluminen todos los entramados sociales que privan a las mujeres de su libertad.

\section{ESTAMOS AQUÍ... IGUAL QUE TÚ}

Donna Haraway acuñó para el conocimiento el símil de un juego de cuerdas (1997) que va rotando de mano en mano realizando figuras diferentes, de situación en situación para crear nuevas figuras de conocimiento. Conocimiento que, como la vida, no es fijo y cualquier imagen que hoy elaboremos será modificada en el curso de la historia por las mujeres y hombres que la encarnen.

Hemos llegado a una de esas figuras del conocimiento donde podemos reconocer, en primer lugar, como un logro la conceptualización de la violencia contra las mujeres como producto del heteropatriarcado. Como segundo logro, que los legisladores y las leyes hayan asumido, muchas veces con grandes resistencias, este paradigma feminista de abordaje para la violencia. Con todo, necesitamos revisar la violencia contra las mujeres, a la luz del neoliberalismo actual y su alianza con el patriarcado, desde la óptica de la privación de libertad que engendra. Asimismo, nos resta por reconocer que usamos toda una serie de términos como violencia de género, violencia machista o violencias feminicidas de forma casi sinónima y nos enfrentamos al reto de pensarlos, acotarlos, desenvolverlos con la finalidad de buscar, en palabras de las epistemólogas, visiones del mundo menos dominadoras. Es decir, más objetivas, atendiendo a la definición de objetividad que asumimos desde la epistemología feminista. Los caminos de futuro, los de la libertad de las mujeres, están llenos de voces, algunas silenciadas en el pasado o, 
incluso, el presente. Voces, diversas y múltiples, para pensar conceptos y categorías, en clave feminista, capaces de despatriarcalizar la política.

Demos voz a las doce criadas:

"cargamos con la culpa

fue injusto

pero ahora estamos aquí

nosotras también estamos aquí

igual que tu."

Margaret Atwood 


\section{REFERENCIAS BIBLIOGRÁFICAS:}

Adán, Carme (2006) Feminismo y conocimiento. A Coruña: Edicións Espiral.

Agra, María Xosé (2013) "Construcciones sociales vinculadas a la violencia de género". En Cristina Alonso (coord.) Violencia de género y Justicia. Santiago de Compostela: Servizo de Publicacións e Intercambio Científico. Campus Vida, pp. 31-42.

Agra, María Xosé (2016) ¿Olvidar a Clitemnestra? Sobre justicia e igualdad. Santiago de Compostela: Servizo de Publicacións e Intercambio Científico. Campus Vida.

Amorós, Celia (1997) Tiempo de feminismo. Madrid: Cátedra.

Amorós, Celia (2008) Mujeres e imaginarios de la globalización. Santa Fe: Homo Sapiens Ediciones.

Atwood, Margariet (2005) Penélope y las doce criadas. Salamandra Ediciones

Beauvoir, Simone de (1989) El Segundo Sexo. Buenos Aires: Siglo veinte.

Butler, Judith (1990) Gender Trouble. New York: Routledge.

Echeverría, Javier (1999) Introducción a la metodología de la ciencia. Madrid: Catedra

Haraway, Donna (1995) Ciencia, Cyborg y mujeres. La reinvención de la naturaleza, Madrid: Cátedra.

Haraway, Donna (1997): Modest_Witness@Second_Millennium.FemaleMan_ Meets_ OncoMouse: Feminism and Technoscience, Londres, Routledge.

Haraway, Donna (1999) "Las promesas de los monstruos: Una política regeneradora para otros inapropiados/bles". Política y Sociedad, 30, pp.121-163

Haraway, Donna (2000) How Like a Leaf: An Interview with Thyrza Nichols Goodeve/ Donna Haraway. Londres: Routledge.

Harding, Sandra (1990) "Feminism, Science, and the Anti-Enlightenment Critiques". En Linda Nicholson (ed.), Feminism/Postmodernism, Londres: Routledge, pp. 83-106.

Harding, Sandra (1991): Whose Science? Whose Knowledge? Ithaca: Cornell University Press.

Harding, Sandra (1993a) Ciencia y Feminismo, Madrid: Morata.

Harding, Sandra (1993b) "Rethinking Standpoint Epistemology: What Is "Strong Objetivity”?”. En L. Alcoff e E. Potter (eds.), Feminist Epistemologies, Londres: Routledge, pp. $49-82$.

Harding, Sandra (1998) Is Science Multicultural?: Postcolonialisms, Feminisms, and Epistemologies. Bloomington: Indiana University Press. 
Hartsock, Nancy (1998) The Feminist Standpoint Revisited and Other Essays, Westview: Oxford.

Hustvedt, Siri (2017) La mujer que mira a los hombres que miran a las mujeres. Barcelona: Seix Barral.

Izquierdo, Ma Jesús (1998) El malestar en la desigualdad. Madrid: Cátedra.

Lagarde, Marcela (2008) "Antropología, feminismo y política: violencia feminicida y derechos humanos de las mujeres". En Margaret Bullen, Carmen Diez Mintegui (coords.) Retos teóricos y nuevas prácticas. Donostia: Ankulegi Antropologia Elkartea. https://www. ankulegi.org/00-retos-teoricos-y-nuevas-practicas.html (Consultado el 05-01-2012)

Longino, Helen (1990): Science as Social Knowledge. N. J.: Princenton University Press.

Longino, Helen (1993a): "Subject, Power and Knowledge: Description and Prescription in Feminist Philosophies of Science". En L. Alcoff y E. Potter (eds.), Feminist Epistemology. Londres: Routledge, pp. 101-120.

Longino, Helen (1990): (1993b) "Essential Tensions- Phase Two: Feminist, Philosophical and Social Studies of Science". En Louise M. Antony y Charlotte Witt (eds.), A Mind of One's Own, Oxford: Westview Press, pp. 257-272.

Longino, Helen (1994) "In Search of Feminist Epistemology". The Monist, 77 (4), pp. 472-485.

Longino, Helen (1997) “Feminismo y filosofía de la ciencia”. En Marta I. González García, José A. López Cerezo e José Luis Luján (eds.), Ciencia, Tecnología y sociedad, Barcelona: Ariel, pp. 71-83.

Lorde, Audre (2004) La hermana, la extranjera. Madrid: Horas y horas

Platero, Lucas R.; Rosón, María y Esther Ortega (coords.) (2017) Barbarismos queer y otras esdrújulas. Barcelona: Bellaterra Edicions.

Rubin, Gayle (1998) "The Traffic in Women". En Linda Nicholson (ed), The Second Wave. A Reader in Feminist Theory, Londres: Routledge, pp. 27-62.

Segato, Rita (2013) La escritura en el cuerpo. México: Tinta limón ediciones.

Segato, Rita (2016) La guerra contra las mujeres. Madrid: Traficante de sueños.

Varela, Nuria (2017) Cansadas, Barcelona: Ediciones B.

Torres, María Concepción (2016) "La violencia de género en la ley integral:consideraciones críticas tras diez años de violencia desde la óptica constitucional". En Teresa San Segundo Manuel (dir.) A vueltas con la violencia. Una aproximación multidisciplinar a la violencia de género. Madrid: Tecnos.

Wollstonecraft, Mary (1994) Vindicación de los derechos de la mujer. Madrid: Cátedra 